\title{
Analytic Biosurveillance Methods for Resource-Limited Settings
}

\author{
Howard Burkom*, Yevgeniy Elbert, Erhan Guven and Jacqueline Coberly \\ Johns Hopkins Applied Physics Laboratory, Laurel, MD, USA
}

\section{Objective}

The authors develop open-source temporal alerting algorithms for data environments characteristic of resource-limited geographic settings and recommend appropriate usage of each.

\section{Introduction}

Biosurveillance in resource-limited settings is essential because of both enhanced risk of diseases rarely seen elsewhere (e.g. cholera) and pandemic threats (e.g. avian influenza). However, access to care and laboratory test capability are typically inadequate in such areas, amplifying the importance of syndromic surveillance [1]. Such surveillance in turn may be a challenge because of insufficient data history and systematic or seasonal behavior. The Suite for Automated Global Electronic bioSurveillance (SAGES) is a collection of modular, freely-available software tools to enable electronic surveillance in these settings [2]. These tools require statistical alerting methods appropriate for SAGES data, and development of such methods is the subject of this effort.

We evaluated alerting methods for two main uses: weekly surveillance for seasonal outbreaks such as dengue fever and influenza, and daily syndromic data for settings where monitoring and response on a daily basis are practical. The latter situation has the added complication that day-of-week clinical visit patterns differ widely, (e.g. clinic closure on Sundays and Thursdays) and may evolve over time.

\section{Methods}

For comparisons using weekly data, we used authentic SAGES time series with effects of seasonal dengue outbreaks covering 19 seasons for 5 provinces. Fewer SAGES data were available on a daily basis, so time series were simulated stochastically to emulate combinations of observed data scales, autocorrelation types, and day-ofweek patterns. For detection performance testing, signals representing outbreak effects were injected into these series with random lognormal draws such that the theoretical outbreak peak was 2 standard deviations above the expected value, with injected counts adjusted for the day-of-week pattern. The methods compared were modifications of standard CUSUM and EWMA charts adapted for SAGES, an adaptation of the CDC EARS C2 algorithm, and GS_SAGES, a method based on generalized exponential smoothing designed to adjust for evolving cyclic behavior without the data requirements of regression models. Open-source code for these methods is available in SAGES. Evaluation criteria included the ratio of injected events detected (event-sensitivity), the delay from the start of injection to alerting (timeliness), the ratio of inject alert days to all inject days (temporal sensitivity, or coherence), the ratio of inject alert days to all alert days (PPV), and one minus the background alert rate (specificity). Recommendations were derived from these measures by choosing the algorithms with least detection delay among all others satisfying numerical criteria for event-sensitivity $(>95 \%)$, coherence $(>25 \%)$, PPV ( $>30 \%)$, and specificity $(>97 \%)$..

\section{Results}

Results: Algorithm recommendations for SAGES users were supplied for the requested situational chart in the Table. Parameters yielding best performance according to above criterion combination will be supplied with code.

\section{Conclusions}

Open-source temporal alerting methods have been developed with recommendations for surveillance use in expected resource-limited data situations. The selection criterion combination was derived from hospital surveillance literature, and numerical component criteria were derived from testing. When disease prevalence is known, the relationship between PPV and coherence, specificity, and prevalence should be used to adjust individual criteria, restricted by local capacity for investigation and response.

Alerting Recommendations by Data Condition

\begin{tabular}{|c|c|c|c|c|}
\hline Data Condition / Method & GS-SAGES & EWMA-SAGES & CUSUM-SAGES & Adapted C2 \\
\hline Daily Data & 1 & 2 & 2 & 2 \\
\hline Monthly Data & 2 & 2 & 1 & 2 \\
\hline >=8 Weeks' Data History & 1 & 1 & 1 & 2 \\
\hline < 8 Weeks' Data History & 2 & 1 & 1 & 2 \\
\hline Sparse Data & 2 & 2 & 1 & 3 \\
\hline Strong Day-of-Week Effect & 1 & 3 & 3 & 3 \\
\hline Strong Seasonal Effect & 1 & 2 & 2 & 2 \\
\hline
\end{tabular}

Codes: $1=$ preferred, $2=$ acceptable, $3=$ not recommended

\section{Keywords}

alerting; control chart; exponential smoothing; SAGES; resourcelimited

\section{Acknowledgments}

The authors acknowledge assistance of the U.S. Armed Forces Health Surveillance Center-Global Emerging Infection System, which made this effort possible.

\section{References}

1. Chretien JP, Burkom HS, Lewis SH, et al. (2009) Syndromic Surveillance: Adapting Innovations to Developing Settings. PLoS Medicine 5(3): e 72

2. Lewis SL, Feighner BH, Loschen WA, et al. (2011) SAGES: A Suite of Freely-Available Software Tools for Electronic Disease Surveillance in Resource-Limited Settings. PLoS ONE 6(5): e19750

\section{*Howard Burkom}

E-mail: howard.burkom@jhuapl.edu 